\title{
Modifications to Internal Tide Conversion Parameterizations and Implementation into Barotropic Ocean Models
}

\author{
William J. Pringle ${ }^{\mathrm{a}, *}$, Damrongsak Wirasaet ${ }^{\mathrm{a}}$, Joannes J. Westerink ${ }^{\mathrm{a}}$ \\ ${ }^{a}$ Environmental Fluid Dynamics Group, Department of Civil and Environmental Engineering and Earth Sciences, \\ University of Notre Dame, Notre Dame, IN, 46656, USA
}

\begin{abstract}
Parameterization of the tidal energy conversion from barotropic to baroclinic modes through internal tide generation over rough and steep submarine topography is a necessary dissipative force for barotropic ocean models. We present and unify two forms of parameterizations that differ by assuming that wave generation is either only due to the local topographic features, or that it includes the effects of nonlocal topographic features. Previously reported implementation schemes on structured grids are extended to unstructured grids. A positive definite modification to the nonlocal generation formulation is recommended to ensure dissipation everywhere. Modifying the buoyancy frequency variable used in the formulations, in order to redistribute some of the dissipation from shallow water into deeper waters, is also proposed. MATLAB functions to compute the necessary parameters on both structured and unstructured grids are highlighted and made available.
\end{abstract}

- Unification of local and nonlocal formulations

- Positive definite dissipation matrix

- Depth-weighted average buoyancy frequencies and nonlocal buoyancy frequencies

- Implementation on unstructured and structured grids

Keywords: unstructured grid, barotropic tides, internal tide generation, topographic gradients, buoyancy frequency

\section{Method Details}

\subsection{Background}

Barotropic ocean models require a parameter to account for the barotropic tidal energy conversion to baroclinic energy through internal tide generation over steep and rough topography in the ocean. It is typically implemented through a linear wave drag type variable so that tidal energy conversion $E_{f}$ is proportional to the tidal velocity squared (Jayne and St. Laurent, 2001):

$$
E_{f}=\rho_{0} h\left\langle\boldsymbol{u}^{T} \mathbf{C u}\right\rangle
$$

\footnotetext{
${ }^{*}$ Corresponding author

Email address: wpringle@nd.edu (William J. Pringle)
} 


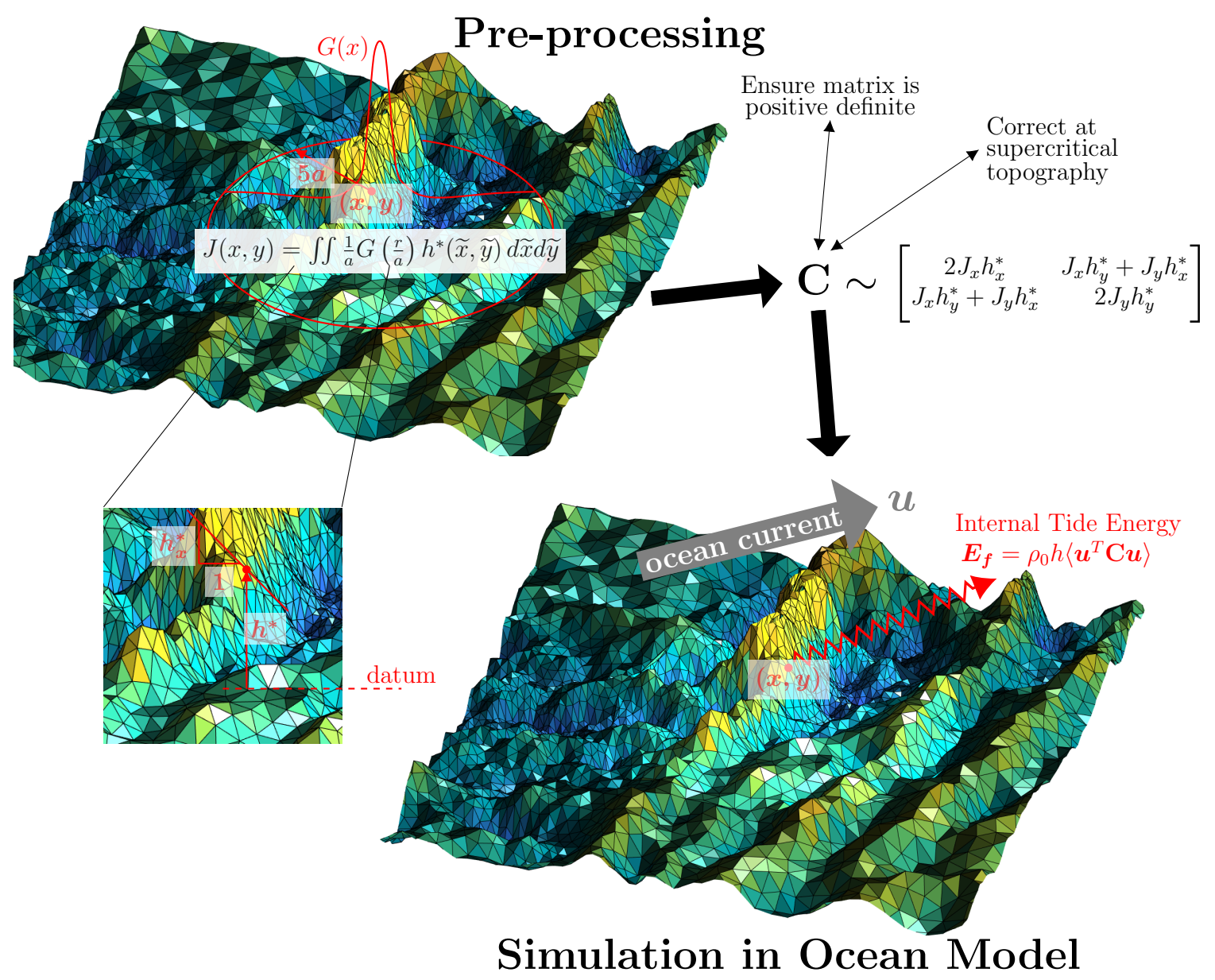

Graphical Abstract: Barotropic ocean currents $\boldsymbol{u}$ generate internal waves over submarine ridges, dissipating barotropic energy, $E_{D}$. A convolution integral of the topographic features, $J$ within the circle drawn is a measure of the propensity for internal wave generation

where \langle\rangle indicates time averaging, $\rho_{0}$ is the reference density of the ocean, $h$ is the topographic depth, $\boldsymbol{u}$ is the depth-averaged velocity vector, and $\mathbf{C}$ is the dissipation matrix that we need to formulate.

Two formulations of $\mathbf{C}$ are outlined in this study that are based on a linear theory solution Bell (1975), valid for subcritical topography $(\gamma<1)$, where:

$$
\begin{aligned}
& \gamma=\frac{\|\nabla h\|}{\alpha} \\
& \alpha=\left(\frac{\omega^{2}-f^{2}}{N_{b}^{2}-\omega^{2}}\right)^{1 / 2}
\end{aligned}
$$

in which $\alpha$ is the internal wave slope, $\omega$ is the angular frequency of the pertinent tidal wave (note that most typically the $\mathrm{M}_{2}$ tidal wave is simply chosen here even when diurnal tides are also present, 
e.g. Pringle et al., 2018), $f$ is the Coriolis frequency, and $N_{b}$ is the Brunt-Väisälä frequency at the seabed. The first formulation assumes that internal wave generation is based only on the local topographic features. The second formulation includes the effect of nonlocal topographic features in the internal wave generation process. The implementation of these formulations on both structured and unstructured grids are outlined. Modifications to the dissipation matrix of the second formulation, and to the buoyancy frequency variable used, in particular when dealing with supercritical topography, are suggested. Algorithms written in MATLAB to compute the equations that are introduced, in addition to the computation of parameters such as the buoyancy frequencies, are briefly described and are made available at the lead author's GitHub website.

\subsection{Local Generation Formulation}

A formulation for $\mathbf{C}$ that is simple to calculate and implement into an ocean model is:

$$
\mathbf{C}=C \frac{\left[\left(N_{b}^{2}-\omega^{2}\right)\left(\omega^{2}-f^{2}\right)\right]^{1 / 2}}{4 \omega \kappa h}\left[\begin{array}{cc}
h_{x}^{2} & h_{x} h_{y} \\
h_{x} h_{y} & h_{y}^{2}
\end{array}\right]
$$

where $C$ is a scale factor (see $§ 1.4$ ), the subscripts ' $x$ ' and ' $y$ ' indicate gradients in the longitudinal and latitudinal directions (in projected map coordinates) respectively, and $\kappa$ is the typical horizontal bathymetric wave number (Lyard et al., 2006). In our version of this method we adopt the idea of Zaron and Egbert (2006) that $\kappa$ is equal to the fundamental internal mode at the pertinent tidal frequency so that $\kappa$ varies depending on the local buoyancy frequency and latitude, i.e.:

$$
\kappa=\frac{\pi}{h}\left(\frac{\omega^{2}-f^{2}}{\tilde{N}^{2}-\omega^{2}}\right)^{1 / 2}
$$

so that (4) rearranges to:

$$
\mathbf{C}=C \frac{\left[\left(N_{b}^{2}-\omega^{2}\right)\left(\tilde{N}^{2}-\omega^{2}\right)\right]^{1 / 2}}{4 \pi \omega}\left[\begin{array}{cc}
h_{x}^{2} & h_{x} h_{y} \\
h_{x} h_{y} & h_{y}^{2}
\end{array}\right]
$$

in which $\tilde{N}$ is the depth-averaged Brunt-Väisälä frequency. Conveniently, (6) is now independent of $h$. Note that the matrix formulation of (6) only dissipates across topographic gradients $\left(h_{x}\right.$ and $h_{y}$ ), but not along them, and it has the property of being positive definite so that it guarantees dissipation at every location.

For implementation in an ocean model, we would like to calculate the topographic gradients $\nabla h$ as accurately as possible, preserving the full topographic roughness of the original bathymetric data. In this case, unless the resolution of the computational grid is similar to the bathymetric grid, the suggested method is to compute the gradients directly on the bathymetric grid before interpolating these onto the computational nodes (c.f. Green and Nycander, 2013). Here, a trick that we find to be useful is interpolating the mean absolute bathymetric gradients within the local computational grid size to the computational node, and multiplying this by the dominant sign of the gradient (implemented by our GridAvInterp MATLAB function that interpolates bathymetry and bathymetric gradients to an unstructured mesh using a cell-averaged technique). This ensures that dissipation, which relies on the topographic gradients, is not biased towards smaller sized computational elements since bathymetry is generally smoothed out over the larger elements.

Typically the bathymetric data is defined on a structured grid, and the gradients can be trivially calculated. If the bathymetric data, for whatever reason, is defined on an unstructured grid, or is a set of scattered points that can be triangulated, an element area-averaged approximation of the slope is usually suitable (see our MATLAB function Unstruc_Bath_Slope). 


\subsection{Nonlocal Generation Formulation}

Nycander (2005) derived a formulation for $\mathbf{C}$ that takes into account the nonlocal generation of internal tides through a convolution integral of the topographic roughness. It has been shown to be marginally superior to other formulations (Green and Nycander, 2013; Pringle et al., 2018). The corresponding matrix $\mathbf{C}$ has the following form in a general coordinate system (Green and Nycander, 2013):

$$
\mathbf{C}=C \frac{N_{b}}{4 \pi h} \sqrt{1-\frac{f^{2}}{\omega^{2}}}\left[\begin{array}{cc}
2 J_{x} h_{x}^{*} & J_{x} h_{y}^{*}+J_{y} h_{x}^{*} \\
J_{x} h_{y}^{*}+J_{y} h_{x}^{*} & 2 J_{y} h_{y}^{*}
\end{array}\right]
$$

where $J$ is a convolution integral of a filtered Green's function of the topographic heights $h^{*}$ (defined positive from the seabed):

$$
J(x, y)=\iint \frac{1}{a} G\left(\frac{r}{a}\right) h^{*}(\widetilde{x}, \widetilde{y}) d \widetilde{x} d \widetilde{y}
$$

where $r=\sqrt{(x-\widetilde{x})^{2}+(y-\widetilde{y})^{2}}$. Within (8) the Green's function, $G(x)$ and filter cutoff length, $a$ are:

$$
\begin{aligned}
G(x) & =\frac{1}{x}-\frac{\sqrt{\pi}}{2} \exp \left(-x^{2} / 8\right) I_{0}\left(x^{2} / 8\right) \\
a & =\frac{\beta}{\pi \sqrt{\omega^{2}-f^{2}}} \tilde{N} h
\end{aligned}
$$

in which $\beta=1.455$ is a numerical coefficient, and $I_{0}$ is the modified Bessel function of the first kind (Nycander, 2005). The gradients of $h^{*}$ can be computed in the same fashion as the gradients of $h$ for the local generation formulation. Also note that for practical purposes $h^{*}=-h$.

The numerical scheme to compute $\nabla J\left(J_{x}\right.$ and $\left.J_{y}\right)$ on a structured grid has been previously detailed (Green and Nycander, 2013). The scheme contains a special treatment of the quadrature rule in the vicinity of a point at which $\nabla J$ is evaluated (more precisely, at the cell corner) in order to reduce the inaccuracy introduced by the singularity in the integrand. We provide an implementation of the scheme in MATLAB (see our function Calc_dJ_Nyc_Struc). However, to compute the convolution integral at every point we are required to search within a cutoff radius up to approximately $a_{c}=5 a$ (where $a$ ranges between $\sim 10-100 \mathrm{~km}$ ) before the Green's function is sufficiently small (Nycander, 2005). As a result, the computation can be very time consuming. In the case of a structured grid we can make use of the knowledge of a constant grid spacing to reduce the searching cost but the overall computation time is still somewhat large. However, because the algorithm is trivially parallelizable, it is possible to break the computation into manageable chunks.

On the other hand, $\nabla J$ in the context of $C^{0}$-finite element method can be calculated directly on an unstructured grid through the use of Gauss quadrature rule for triangles. More specifically, the gradient of $J$ in the $x$-direction at a given finite element node $i,\left(x_{i}, y_{i}\right)$,

$$
J_{x}\left(x_{i}, y_{j}\right)=\iint F_{i}(\widetilde{x}, \widetilde{y}) \frac{x_{i}-\widetilde{x}}{r_{i}} d \widetilde{x} d \widetilde{y}, \quad F_{i}(\widetilde{x}, \widetilde{y}) \equiv \frac{1}{a} h^{*}(\widetilde{x}, \widetilde{y}) G^{\prime}\left(\frac{r_{i}}{a}\right)
$$

is approximated by:

$$
J_{x} \approx \sum_{e \in \mathcal{T}_{a}} \sum_{j=1}^{M_{c}} w_{c, j} F_{i}\left(\widetilde{x}_{c, j}^{e}, \widetilde{y}_{c, j}^{e}\right)
$$

where $\mathcal{T}_{i, a}$ is a set of triangle elements within the area in which $r_{i}<a_{c},\left\{\left(x_{c, j}^{e}, y_{c, j}^{e}\right)\right\}$ and $\left\{w_{c, j}\right\}$ are the quadrature points and weights associated with an element $e$, and $M_{c}$ is the number of quadrature points. The gradient of $J$ in the $y$-direction can be approximated in a similar manner. In this work, 
we use quadrature rules devised for integration over a triangle (Dunavant, 1985; Hesthaven and Warburton, 2008). Such quadrature rules contain only points in the interior of the triangle; thus, the formula (11) does not involve the singularity at $\left(x_{i}, y_{i}\right)$. Here, we use the quadrature rule integrating exactly cubic polynomials in the calculations. Note that although this quadrature rule is locally fourth-order accurate, the singularity in the integrand of (11) limits the global order of accuracy of the formula (12) to first-order.

The implementation of the scheme on an unstructured grid in MATLAB is provided (see our function $C a l c_{-} d J_{-} N y c_{-} U n s t r u c$ ). It can be advantageous to calculate $\nabla J$ on unstructured grids because often elements are larger in deeper waters and smaller in shallow high gradient regions. Since $a$ and thus the cutoff search radius, $a_{c}$ is proportional to $h$, the scheme can become more computationally efficient than on a structured grid because the number of elements in the search radius may remain fairly constant with depth.

\subsubsection{Positive Definite Modification}

The form of $\mathbf{C}$ in (7) has been rotated from the major and minor axis of the tidal ellipse used in the original formulation (Nycander, 2005). As a result, $\mathbf{C}$ is not guaranteed to be positive definite when the sign of the gradients of $J$ and $h^{*}$ in the $x-y$ coordinate system do not conform. This is in general an undesired quality since the positivity of energy dissipation is not ensured for a non-positive definite $\mathbf{C}$. Thus, we propose to modify $\mathbf{C}$ at the computational nodes where it has a negative eigenvalue by finding the nearest positive definite matrix to $\mathbf{C}$ (Higham, 1988) (see our MATLAB function Eigen_neg_to_pos). This positive definite modification has been shown to give slightly improved results from the original formulation (Pringle et al., 2018).

\subsection{Suggested Values of the Scale Factor}

The scale factor in the two formulations is necessary for real ocean applications of numerical models. The optimal value of $C$ for the local generation formulation was found to be equal to 2.76 in Pringle et al. (2018) for their study in the Indian and Western Pacific Oceans (NB: in that study $C$ was not divided by $4 \pi$ hence was quoted as being equal to 0.22 ). Similarly, the optimal value of $C$ for the nonlocal generation formulation was found to be equal to approximately 2.75 in Buijsman et al. (2015) for their global model, and 2.95 in (Pringle et al., 2018). So both methods and the two different studies quoted appear to suggest some convergence of the scale factor. Note that as far we as understand, this study is the first to unify the local and nonlocal formulations and their scale factors. It is worth mentioning that the value of $C$ (2.75 to 3.0) is not necessarily a universal number. In particular, Buijsman et al. (2015) show that in the Atlantic Ocean $C=5$ would result in a smaller integrated error, which agrees with the authors' very recent experience in modeling the North Atlantic.

\subsection{Supercritical Topography Correction and Modifications to the Buoyancy Frequency Variable}

Since the parameterizations are both only valid for subcritical topography, it is typical to assume that the dissipation becomes saturated for supercritical topography $(\gamma>1)$ (Melet et al., 2013; Buijsman et al., 2015). In other words, $\mathbf{C}$ is divided by $\gamma^{2}$ when $\gamma>1$. It is at least partly because of this correction that a scale factor even for the nonlocal generation formulation is required.

It has been shown that the formulations of $\mathbf{C}$ presented in this study are more dissipative in shallow waters and less in deeper waters within forward barotropic models than data-assimilated ones (Pringle et al., 2018; Buijsman et al., 2015). Whether or not this is a major issue, the definition of the buoyancy frequency can be adjusted to move some of the dissipation into deeper waters. For example, $N_{b}$ could be replaced by $\tilde{N}_{w}$, "a depth-weighted average buoyancy frequency, with weights decreasing linearly from the bottom up to the surface" (Lyard et al., 2006). It is important to note 

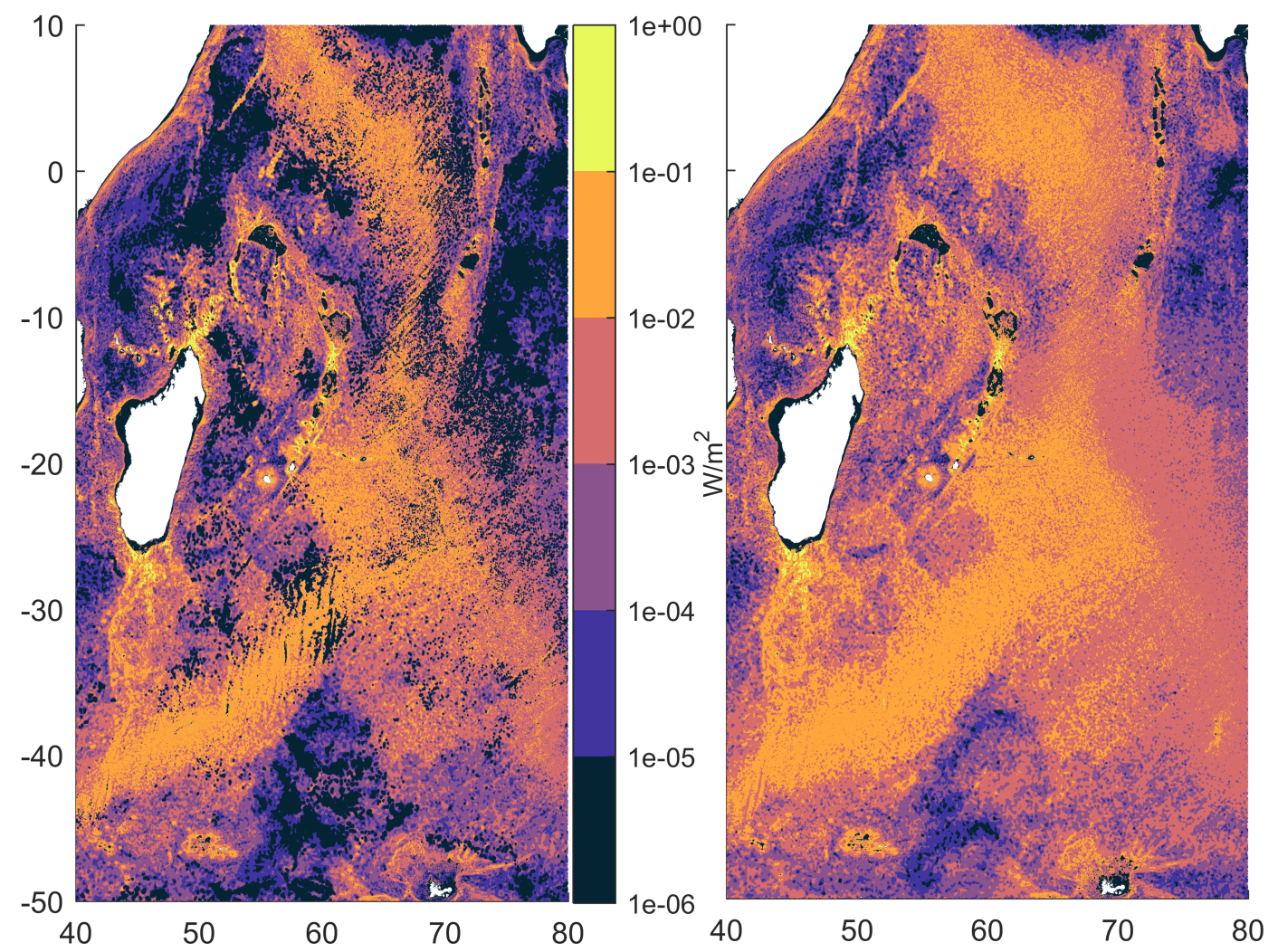

Figure 2: Comparison of the internal tide energy conversion (1) of the $\mathrm{M}_{2}$ tidal wave over the Western Indian Ocean Ridge when using $N_{b}$ (left), and when replacing it with $\tilde{N}_{w}$ (right) in (3) and (6), when using the local generation formulation for $\mathbf{C}$

that generally, $\tilde{N}_{w}>N_{b}$. Thus, because $\alpha \sim 1 / N_{b}(3)$ and $\gamma \sim 1 / \alpha(2), \gamma$ is increased when $\tilde{N}_{w}$ is used instead of $N_{b}$. As a result, more topography would be classified as supercritical and saturated out. Furthermore, since $\mathbf{C} \sim N_{b}$ in (6) and (7), the average magnitude of $\mathbf{C}$ will increase when $\tilde{N}_{w}$ replaces $N_{b}$. This generally implies that deeper regions with smaller topographic gradients become more dissipative, and shallower high gradient regions become less dissipative. The effect on the spatial distribution of internal tide energy conversion (1), is that it will be more evenly spread out over ocean ridges and other topographical features (Fig 2).

With regards to the nonlocal generation formulation, the effect of the nonlocal buoyancy frequencies may be important in the same way that the nonlocal topographic features are. Thus, we propose Gaussian smoothing of $N_{b}$ scaled by $a^{2}$ :

$$
N_{b a v}=\frac{\iint e^{-\frac{r^{2}}{a^{2}}} N_{b}(\widetilde{x}, \widetilde{y}) d \widetilde{x} d \widetilde{y}}{\iint e^{-\frac{r^{2}}{a^{2}}} d \widetilde{x} d \widetilde{y}}
$$

in which the integral can be truncated at a cutoff search radius, $a_{c} \approx 2.5 a . N_{b a v}$ is the Gaussian smoothed buoyancy frequency at the seabed to replace $N_{b}$ in (3) and (7). Its computation is implemented in our Compute_Nb_greens_av MATLAB function. Note that the optimal scale factor $C$ may be slightly different than when $N_{b}$ is used. The modification to use $N_{b a v}$ instead of $N_{b}$ for the 
nonlocal generation formulation has the effect of reducing some of the dissipation in waters shallower than approximately $2500 \mathrm{~m}$ and increasing dissipation in depths greater than this in the Indian and Western Pacific Oceans (Pringle et al., 2018).

Be aware that using $\tilde{N}_{w}$ and $N_{b a v}$ instead of $N_{b}$ in the local and nonlocal generation formulations respectively, is simply an option that users might employ to improve the distribution of dissipation if required for their application. In particular, using $\tilde{N}_{w}$ in the local generation formulation can change the distribution of dissipation quite substantially compared to $N_{b}$ (Fig 2), thus it is not necessarily a recommended option. The effects of using $N_{b a v}$ in the nonlocal generation formulation appear to be much milder. Initially, for both formulations using $N_{b}$ is probably a good idea.

\subsection{Calculation of Buoyancy Frequencies}

The Brunt-Väisälä buoyancy frequency information required for both formulations can be calculated from e.g., gridded World Ocean Atlas 2013 data of salinity (Zweng et al., 2013) and temperature (Locarnini et al., 2013), or from a 3D baroclinic ocean model output. The GSW Oceanographic Toolbox (McDougall and Barker, 2011) is used to convert salinity and temperature into vertical profiles of the buoyancy frequency $N$ defined on the grid of the original data (see our MATLAB function Make_Gridded_ $N$ which is programmed to compute $N$ using the World Ocean Atlas 2013 in NetCDF format).

To obtain the values of $N_{b}, \tilde{N}$, and $\tilde{N}_{w}$ at the computational nodes of the grid (implemented in our MATLAB function Gridded_to_Mesh_SeaBed_DepthAveraged), firstly $N$ is interpolated onto the computational grid at every depth contour less than the depth of the computational node, $h . N_{b}$ is found by linear interpolation of the vertical profile of $N$ at $h . \tilde{N}$ is evaluated by the following:

$$
\tilde{N}=\frac{\sum_{k} N_{k} \Delta z_{k}}{h} \quad \forall z_{k}<h
$$

where $z_{k}$ is the depth contour (positive downwards), and $\Delta z_{k}=\min \left(z_{k+1}-z_{k}, h-z_{k}\right) . \quad \tilde{N}_{w}$ is evaluated in the same fashion except with weights applied:

$$
\tilde{N}_{w}=\frac{\sum_{k} w_{k} N_{k} \Delta z_{k}}{h} \quad \forall S D_{k}<h
$$

where $w_{k}=\min \left[0.5\left(z_{k}+z_{k+1}\right) / h, 0.5\left(z_{k}+h\right) / h\right]$.

\section{Acknowledgements}

We thank Dr. Patrick Timko from Bangor University for providing the SRTM30_PLUS bathymetry with synthetic abyssal hill roughness to aid in the testing of the methods presented here. This work was supported by the Office of Naval Research under grant N00014-15-1-2623.

\section{Availability}

The MATLAB functions mentioned to implement the methods described in this study, along with their dependencies, are available at the lead author's GitHub website: https://github.com/ WPringle/ADCIRC_MATLAB/tree/master/Internal_tide. 


\section{References}

Bell, T.H., 1975. Topographically generated internal waves in the open ocean. Journal of Geophysical Research 80, 320-327. URL: http://doi.wiley.com/10.1029/JC080i003p00320, doi:10.1029/ JC080i003p00320.

Buijsman, M., Arbic, B., Green, J., Helber, R., Richman, J., Shriver, J., Timko, P., Wallcraft, A., 2015. Optimizing internal wave drag in a forward barotropic model with semidiurnal tides. Ocean Modelling 85, 42-55. doi:10.1016/j.ocemod.2014.11.003.

Dunavant, D.A., 1985. High Degree Efficient Symmetrical Gaussian Quadrature Rules for the Triangle. International Journal for Numerical Methods in Engineering 21, 1129-1148. doi:10.1002/ nme.1620210612.

Green, J.A.M., Nycander, J., 2013. A Comparison of Tidal Conversion Parameterizations for Tidal Models. Journal of Physical Oceanography 43, 104-119. URL: http://journals.ametsoc.org/ doi/abs/10.1175/JPO-D-12-023.1, doi:10.1175/JPO-D-12-023.1.

Hesthaven, J.S., Warburton, T., 2008. Nodal Discontinuous Galerkin Methods: Algorithms, Analysis, and Applications. volume 54 of Texts in Applied Mathematics. Springer New York, New York, NY. URL: http://link. springer.com/10.1007/978-0-387-72067-8, doi:10.1007/ 978-0-387-72067-8.

Higham, N.J., 1988. Computing a nearest symmetric positive semidefinite matrix. Linear Algebra and its Applications 103, 103-118. URL: http://www.sciencedirect.com/science/article/ pii/0024379588902236, doi:10.1016/0024-3795(88)90223-6.

Jayne, S.R., St. Laurent, L.C., 2001. Parameterizing tidal dissipation over rough topography. Geophysical Research Letters 28, 811-814. URL: http://doi.wiley.com/10.1029/2000GL012044, doi:10.1029/2000GL012044.

Locarnini, R.A., Mishonov, A.V., Antonov, J.I., Boyer, T.P., Garcia, H.E., Baranova, O.K., Zweng, M.M., Paver, C.R., Reagan, J.R., Johnson, D.R., Hamilton, M., Seidov, D., 2013. World Ocean Atlas 2013. Vol. 1: Temperature. Technical Report. URL: https://data.nodc.noaa.gov/woa/ WOA13/DOC/woa13\{_\}Vol1.pdf.

Lyard, F., Lefevre, F., Letellier, T., Francis, O., 2006. Modelling the global ocean tides: modern insights from FES2004. Ocean Dynamics 56, 394-415. URL: http://link.springer.com/10. 1007/s10236-006-0086-x, doi:10.1007/s10236-006-0086-x.

McDougall, T.J., Barker, P.M., 2011. Getting started with TEOS-10 and the Gibbs Seawater (GSW) Oceanographic Toolbox. SCOR/IAPSO WG127.

Melet, A., Nikurashin, M., Muller, C., Falahat, S., Nycander, J., Timko, P.G., Arbic, B.K., Goff, J.A., 2013. Internal tide generation by abyssal hills using analytical theory. Journal of Geophysical Research: Oceans 118, 6303-6318. URL: http://doi.wiley.com/10.1002/2013JC009212, doi:10.1002/2013JC009212.

Nycander, J., 2005. Generation of internal waves in the deep ocean by tides. Journal of Geophysical Research 110, C10028. URL: http://doi.wiley.com/10.1029/2004JC002487, doi:10.1029/2004JC002487. 
Pringle, W.J., Wirasaet, D., Suhardjo, A., Meixner, J., Westerink, J.J., Kennedy, A.B., Nong, S., 2018. Finite-Element Barotropic Model for the Indian and Western Pacific Oceans: Tidal ModelData Comparisons and Sensitivities. Ocean Modelling URL: https://linkinghub.elsevier. com/retrieve/pii/S146350031830026X, doi:10.1016/j.ocemod.2018.07.003.

Zaron, E.D., Egbert, G.D., 2006. Estimating Open-Ocean Barotropic Tidal Dissipation: The Hawaiian Ridge. Journal of Physical Oceanography 36, 1019-1035. URL: http://journals ametsoc. org/doi/abs/10.1175/JP02878.1, doi:10.1175/JP02878.1.

Zweng, M.M., Reagan, J., Antonov, J., Mishonov, A., Boyer, T., Garcia, H., Baranova, O., Johnson, D., Seidov, D., Bidlle, M., 2013. World Ocean Atlas 2013, Volume 2: Salinity. Technical Report. URL: https://data.nodc.noaa.gov/woa/WOA13/DOC/woa13\{_\}vol2.pdf. 